\title{
CARF, As An Oncogene, Promotes Colorectal Cancer Stemness By Activating ERBB Signaling Pathway
}

This article was published in the following Dove Press journal: OncoTargets and Therapy

\author{
Weiyi Dong ${ }^{1, *}$ \\ Zheng $\mathrm{CaO}^{2, *}$ \\ Yanmin Pang ${ }^{3}$ \\ Teng Feng' \\ Hongtao Tian' \\ 'Department of Pathology, Heze \\ Municipal Hospital, Heze City, Shandong \\ 27403I, People's Republic of China; \\ ${ }^{2}$ Department of Pathology, Juye County \\ People's Hospital, Heze City, Shandong \\ 274900, People's Republic of China; \\ ${ }^{3}$ Department of Intensive Care Unit, \\ Heze Municipal Hospital, Heze City, \\ Shandong 27403I, People's Republic of \\ China
}

*These authors contributed equally to this work
Correspondence: Hongtao Tian

Department of Pathology, Heze Municipal Hospital, No. 2888, Caozhou West Road,

Heze City, Shandong 27403 I, People's

Republic of China

Tel +8615553007892

Fax +8605305613311

Email tianhongtao20II@163.com
Introduction: The role of CARF, a calcium-responsive transcription factor, in colorectal cancer initiation and development is still unknown. Here, we report that CARF promotes colorectal cancer stemness through ERBB signaling pathway.

Materials and methods: Both colorectal cancer cell lines and primary cells were used in this study. The levels of target mRNA and protein in the cells were examined by qRTPCR and Western blot. Gene manipulation was achieved by the lentivirus delivery system. Luciferase reporter gene assay was employed to analyze the transcriptional activity of the promoter. ChIP assay was performed for the examination of the binding between CARF and the promoters of MAPK8 and JUN. Kaplan-Meier survival curve was generated by the R2 program. Correlation analysis was performed using Spearman correlation analysis.

Results: Aberrant upregulation of CARF has been found in tumor tissues of colorectal cancer patients and associated with poor prognosis. Ectopic expression of CARF promoted the sphere-formation activities, as well as the expression of stem cell markers in colorectal cancer cells and knockdown of CARF, inhibited these activities. The mechanistic analysis showed that CARF directly binds to the promoter of MAPK8 and JUN, promotes the expression of MAPK8 and JUN, activates the ERBB signaling pathway, and thereby promotes the maintenance of the stemness in colorectal cancer cells.

Conclusion: CARF, as an oncogene, promotes colorectal cancer stemness by activating ERBB signaling pathway. The ERBB signaling pathway that serves as the main downstream effector of CARF could be an efficient drug target for colorectal cancer caused by aberrant expression of CARF.

Keywords: CARF, colorectal cancer, stemness, ERBB signaling, JUN

\section{Introduction}

Colorectal cancer is a leading cause of cancer death, accounting for around 600,000 patients die worldwide, with its incidence increasing year by year. ${ }^{1}$ The standard CRC treatment consists of surgery, targeted therapy, radiotherapy and chemotherapy. $^{2}$ Unfortunately, most anticancer drugs are cytotoxic drugs without exact knowledge of their mechanism of action. ${ }^{3}$ Recently, targeted therapies have been developed on the basis of a particular known molecular target and their abilities to interfere with exact known cellular processes. ${ }^{4}$ However, our understanding of cancer initiation, progression and treatment resistance still needs to be improved to develop better targeted therapeutic strategies. 
Calcium ions $\left(\mathrm{Ca}^{2+}\right)$ serve as a second messenger that plays important roles in fundamental cellular physiology, including gene expression, cell cycle, autophagy and apoptosis. $\mathrm{Ca}^{2+}$ activates downstream signaling through either release from intracellular organelles or influx from extracellular reservoir. ${ }^{5-8}$ The downstream effectors of intracellular $\mathrm{Ca}_{2}^{+}$oscillations, the most important $\mathrm{Ca}^{+}$signal forms, include transcription factors, such as nuclear factor$\kappa \mathrm{B}(\mathrm{NF}-\kappa \mathrm{B})$ and nuclear factor of activated T-cells (NFAT), and kinase, such as calmodulin-dependent protein kinase II (CaMKII), as well as other functional proteins, including calmodulin $(\mathrm{CaM})$ and calpain. ${ }^{8-11}$ Alteration of $\mathrm{Ca}^{2+}$ signaling has been found in cancer cells. For example, induction of proliferation signals by intracellular $\mathrm{Ca}^{2+}$ oscillations has been found in esophageal cancer cells. ${ }^{12}$ Thus, $\mathrm{Ca}^{2+}$ signal could be a therapeutic target for cancer prevention.

Calcium-response factor (CARF) is a transcription factor that was known as a binding protein for the promoter of the gene encoding brain-derived neurotrophic factor (Bdnf). ${ }^{13-15}$ In neurons, $\mathrm{CARF}$ is regulated by $\mathrm{Ca}^{2+}$ influx and activates transcription of BDNF in a CaRE1 (calcium-response element 1)-dependent manner. In addition, the studies of $C A R F$ knockout (KO) mice and genome-wide analysis of CARF binding sites suggested that CARF-induced reprograms of gene transcription may be important for neural development. ${ }^{16,17}$ These facts also suggest the potential role of CARF in cancer initiation and development. However, the role of CARF in cancer initiation and development is still unknown.

In this study, we showed that CARF is aberrantly upregulated in colorectal cancer cells and promotes the maintenance of the stemness of colorectal cancer stem cell through the ERBB signaling pathway. Our study thus, for the first time, revealed the relationship between CARF and the stemness of cancer stem cells.

\section{Materials And Methods}

\section{Cell Culture}

Human colorectal cancer cell lines, HCT-116 and HT-29 were purchased from Cell Bank of Chinese Academy of Sciences, Shanghai. All the cells were cultured in complete DMEM with $10 \%$ fetal bovine serum (FBS) and $1 \%$ penicillin/streptomycin (Invitrogen). For primary cell culture, tissue samples were collected from patients with colorectal cancer undergoing surgical resection of their primary tumor. The tissues were placed in cold phosphate-buffered saline (PBS) containing antibiotics $(500 \mathrm{U} / \mathrm{mL}$ penicillin, $500 \mu \mathrm{g} / \mathrm{mL}$ streptomycin, $100 \mu \mathrm{g} / \mathrm{mL}$ gentamicin and 2.5 $\mu \mathrm{g} / \mathrm{mL}$ amphotericin B) and visible fatty and necrotic areas were removed. The tissues were then minced into 1-2 $\mathrm{mm}$ pieces followed by digestion with $1 \mathrm{mg} / \mathrm{mL}$ collagenase type II (Thermo Fisher Scientific) in PBS for $20 \mathrm{~min}$ at $37^{\circ} \mathrm{C}$. After filter through a $30 \mu \mathrm{m}$ filter, the isolated fragments were seeded in complete DMEM and cultured at $37^{\circ} \mathrm{C}$ in a $5 \%$ $\mathrm{CO} 2$ humidified incubator. The tissues were obtained from Heze Municipal Hospital, which was approved by the ethics committee of Heze Municipal Hospital. The information of the patients is provided in Supplementary Table 1. All patients provided written informed consent, and that this was conducted in accordance with the Declaration of Helsinki. For cancer stem cell isolation, the cells were seeded in stem cell medium (Thermo Fisher Scientific) in Ultra-low adherent plate (Corning) and cultured at $37^{\circ} \mathrm{C}$ in a $5 \% \mathrm{CO} 2$ humidified incubator.

\section{Quantitative Real-Time Reverse- Transcription PCR (qRT-PCR)}

Total RNA was isolated using Trizol reagent (Invitrogen). Complementary DNA was synthesized by the High Capacity cDNA Reverse Transcription Kit (Thermo Fisher Scientific). The Power SYBR Green PCR Master Mix (Thermo Fisher Scientific) was used and the samples were amplified by the 7300 Realtime PCR System (Applied Biosystems). Primers are listed in Supplementary Table 2.

\section{Genetic Manipulation}

Full-length cDNA of CARF was cloned into pCDH plasmid and shRNAs specifically against CARF and JUN (Supplementary Table 3) were cloned into PLKO.1 plasmid. Lenti-X packaging kit (Clontech) was used for lentivirus generation according to the manual. The media with lentivirus particles was used to infect the cells. Stable cell lines were selected by puromycin for 1-2 weeks. The efficiency of genetic manipulation was determined by qRT-PCR.

\section{Antibody And Reagents}

The information on the antibodies is listed in Supplementary Table 4. All other reagents were obtained from Sigma-Aldrich.

\section{Sphere Formation Assay}

One thousand cells were seeded in stem cell medium (Thermo Fisher Scientific) in Ultra-low adherent plate (Corning) and cultured at $37^{\circ} \mathrm{C}$ in a $5 \% \mathrm{CO} 2$ humidified 
incubator for 10-15 days. The spheroids were then observed and counted under a phase-contract microscopy (Leica).

\section{Luciferase Reporter Assay}

MAPK8 and JUN promoters were amplified from the genomic DNA of HCT-116 cells and were cloned into PGL 4.20 luciferase reporter plasmid (Promega). The primers were listed in Supplementary Table 2. The reconstructed plasmid was transfected into the target cells together with hRluc/TK vector control. Dual-Luciferases Reporter Assay kit (Promega) was used to measure the luciferase activity.

\section{Chromatin Immunoprecipitation Quantitative PCR (ChIP-qPCR)}

EpiTect ChIP qPCR Assay kit (Qiagen) was used for ChIP-qPCR assay. Briefly, the chromatin was isolated from fixed cells and was sonicated to an average size of $0.8-1.0 \mathrm{~kb}$. The sample was then incubated with beads with the antibody for immunoprecipitation. The precipitated DNA was then subjected to qRT-PCR analysis. DNA Enrichment was calculated as a percentage of input DNA.

\section{Xenograft Model}

All animal experiments were approved by the Institutional Animal Care and Use Committee (IACUC) at Heze Municipal Hospital. The cells were injected into 4-weekold female athymic nude mice. Kinetic of the tumor was evaluated every 7 days.

\section{Bioinformatics Analysis}

The gene expression data were downloaded from R2: Genomics Analysis and Visualization platform (http://r2. amc.nl). Kaplan-Meier survival curve was generated by the R2 program. Correlation analysis was performed using Spearman correlation analysis.

\section{Statistical Analysis}

All experiments were repeated at least three times. Data were expressed as the mean \pm s.d. The significance was determined by Student's $t$-test (unpaired, two-tailed), $* P<0.05, * * P<0.01, * * * P<0.001$.

\section{Results}

\section{CARF Is Upregulated In Colorectal Cancer} Stem Cells And Predicts Poor Prognosis

As the clinical significance of CARF expression in colorectal cancer patients is still unknown, we first downloaded the gene expression dataset from R2 Genomics Analysis and Visualization platform and performed Kaplan-Meier analysis to investigate the relationship between the expression of CARF and overall survival of colorectal cancer patients. As shown in Figure 1A, patients with higher CARF levels exhibited worse overall survival. Next, the expression of CARF in 160 (two cohorts) colorectal cancer tissues and their adjacent normal tissues were examined by qRT-PCR to investigate whether the expression of CARF is upregulated in colorectal cancer tissues. Indeed, as shown in Figure 1B, the expression of CARF is upregulated in colorectal cancer tissues. These results suggested that CARF may serve as an oncogene in colorectal cancer development.

Next, to investigate whether the expression of CARF is correlated with the stemness of colorectal cancer stem cells, we isolated the colorectal cancer stem cells by flow cytometry and suspension culture, respectively. As shown in Figure $1 \mathrm{C}$ and $\mathrm{D}$, the expression of CARF is upregulated in CD44+ cells (Figure 1C) and spheres (Figure 1D) isolated from both primary cells and cell line cells (HCT116 and HT-29). Subsequently, the correlation between the expression of CARF and CD44 or CD133 was examined in colorectal cancer tissues was examined. As shown in Figure 1E, the expression of CARF is positively correlated with CD44 and CD133. These results demonstrated that CARF is upregulated in colorectal cancer stem cells and indicated that CARF may serve as an oncogene that promotes the stemness of colorectal cancer stem cells.

\section{Ectopic Expression Of CARF Promotes The Stemness Of Colorectal Cancer Stem Cells}

Next, the expression of CARF in colorectal cancer cells, including primary cells and cell line cells, was examined. As shown in Figure 2A, CARF-high and CARF-low colorectal cancer cells were identified. To confirm that CARF promotes the stemness of colorectal cancer stem cells, we next overexpressed CARF in CARF-low colorectal cancer cells (\#1 and LoVo) (Supplementary Figure 1). As shown in Figure $2 \mathrm{~B}$, the overexpression of CARF increased the diameter of the spheres. In addition, ectopic expression of CARF promoted sphere-formation activities in colorectal cancer cells (Figure 2C). Moreover, as shown in Figure 2D, the expression of cancer stem cell markers (CD44 and CD133) was elevated by CARF overexpression. Furthermore, CARF overexpression attenuated the sensitivity of the cells to chemotherapeutic drugs (Figure 2E). As expected, CARF 
A

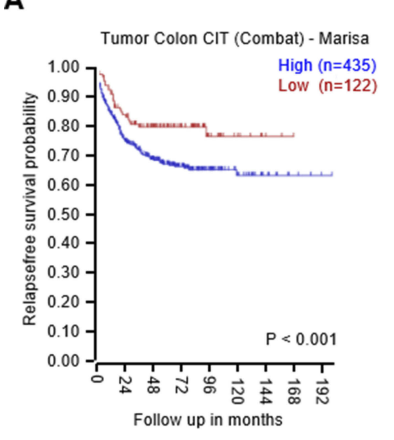

B

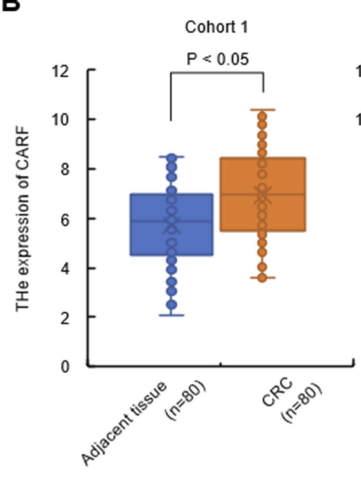

D

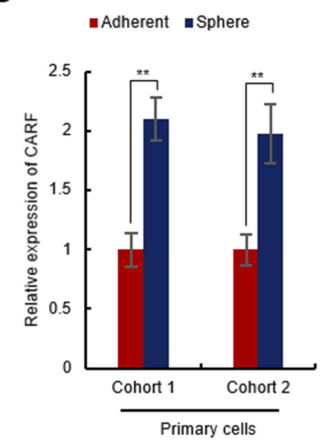

- Adherent $\mathbf{m}$ Sphere

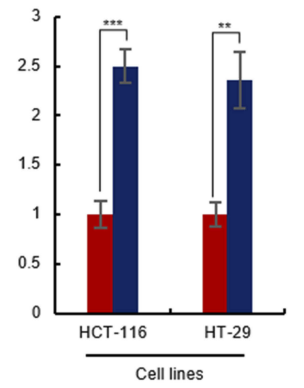

C

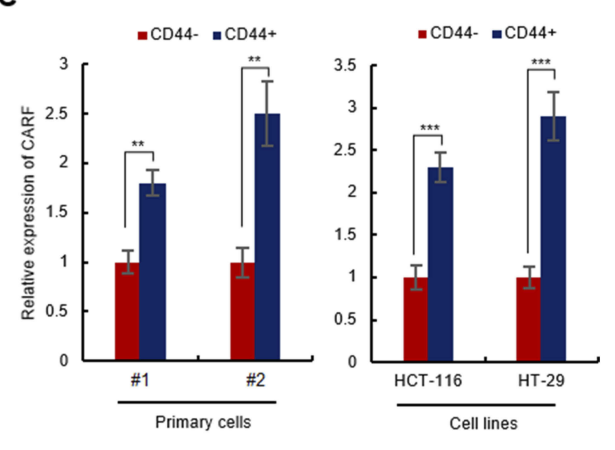

E
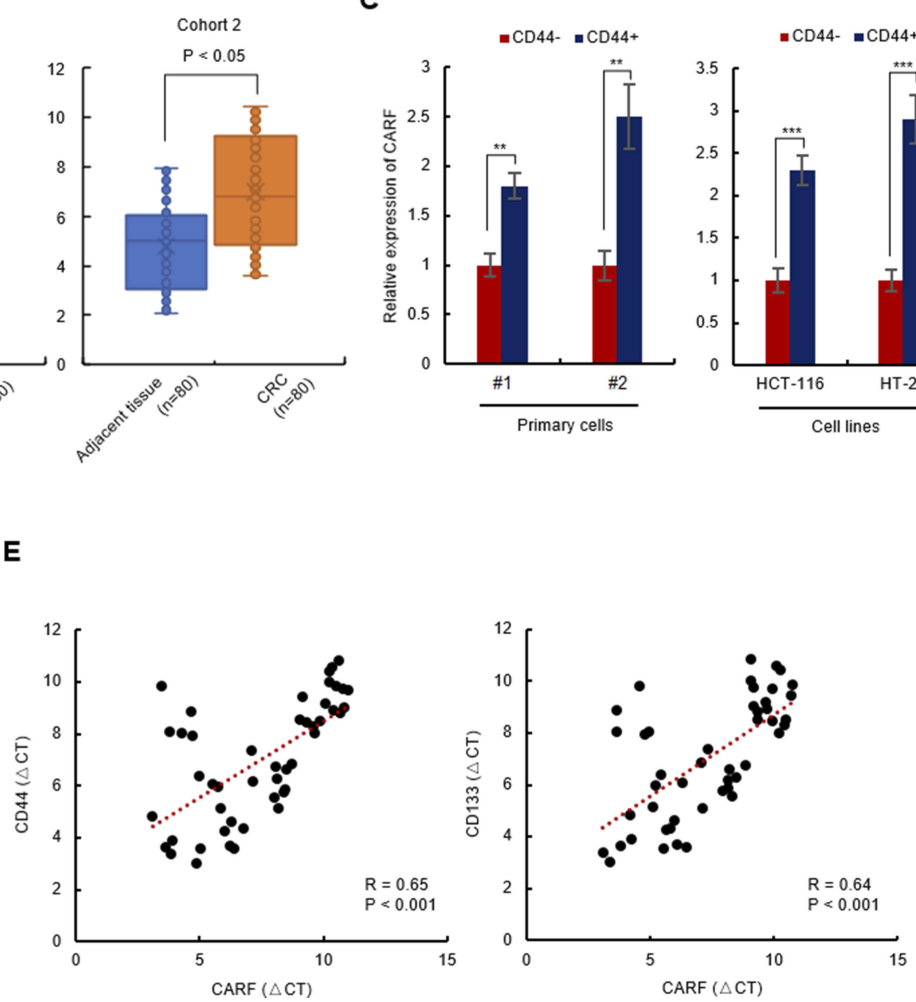

Figure I CARF is upregulated in colorectal cancer stem cells and predicts poor prognosis.

Notes: (A) High expression of CARF predicts poor prognosis. Gene expression data were downloaded from the R2 genomics analysis and visualization platform. KaplanMeier analysis was performed. (B) The expression of CARF was upregulated in colorectal cancer tissue. The mRNA levels of CARF in colorectal cancer tissues and adjacent normal tissues were analyzed by qRT-PCR. (C) The expression of CARF was upregulated in CD44+ colorectal cancer cells. The mRNA levels of CARF in CD44+ and CD44cells isolated from both primary cells and colorectal cancer cell line cells were analyzed by qRT-PCR. (D) The expression of CARF was upregulated in colorectal cancer spheres. The mRNA levels of CARF in colorectal cancer spheres isolated from both primary cells and colorectal cancer cell line cells were analyzed by qRT-PCR. (E) Correlation of CD44 or CDI33 and CARF. The total RNA was isolated from colorectal cancer tissues. The expression levels of CD44, CDI33 and CARF were examined by qRT-PCR and Spearman correlation analysis was performed. (**P $<0.01$, $* * * P<0.001$ ).

overexpression promoted tumorigenesis of the cells in vivo (Figure 2F). These results demonstrated that the ectopic expression of CARF promotes the stemness of colorectal cancer stem cells.

\section{Knockdown Of CARF Inhibits The Stemness Of Colorectal Cancer Stem Cells} To further confirm the role of CARF in the maintenance of colorectal cancer stemness, CARF was depleted in CARFhigh colorectal cancer cells by lentivirus delivery system (Supplementary Figure 2). As expected, knockdown of CARF decreased the sphere-formation activities in both primary cells and cell line cells (Figure 3A and B). Moreover, CARF depletion downregulated the expression of cancer stem cells markers (CD44 and CD133) as indicated by qRT-PCR assay (Figure 3C). Furthermore, CARF knockdown sensitized colorectal cancer cells to chemotherapeutic drugs (Figure 3D). These results indicated that knockdown of CARF inhibits the stemness of colorectal cancer stem cells. Collectively, the above results demonstrated that CARF serves as an oncogene that promotes the stemness of colorectal cancer stem cells.

\section{CARF Activates ERBB Signaling Pathway In Colorectal Cancer Cells}

To investigate the mechanisms underlying CARF promoting the stemness in colorectal cancer cells, we first identified the target signaling pathways of CARF by bioinformatic methods. We downloaded the gene expression dataset from R2 Genomics Analysis and Visualization platform and CARF correlated genes were identified by Spearman analysis. As shown in Figure 4A, we found that the expression of MAPK8 and JUN in the ERBB signaling pathway was positively correlated with CARF. To confirm this result, we performed qRT-PCR to test the mRNA expression of MAPK8 and JUN in CARF-overexpressing colorectal cancer cells. As expected, the expression of MAPK8 and JUN was significantly upregulated in CARF-overexpressing cells (Figure 4B). In addition, the results of the Western blot assay indicated that 
A

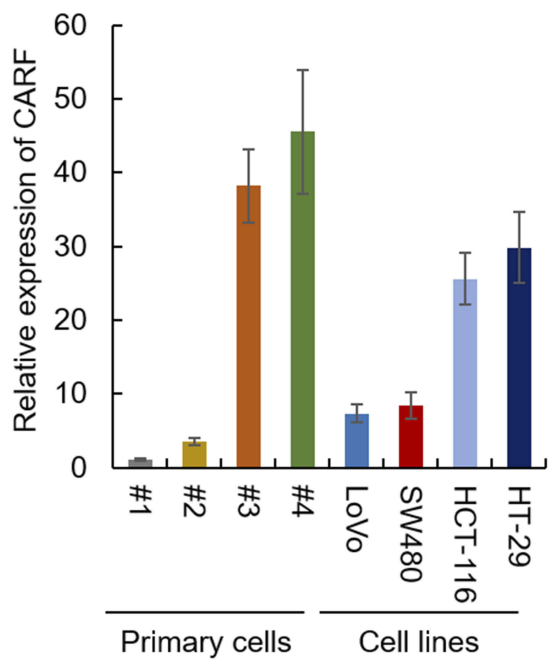

B

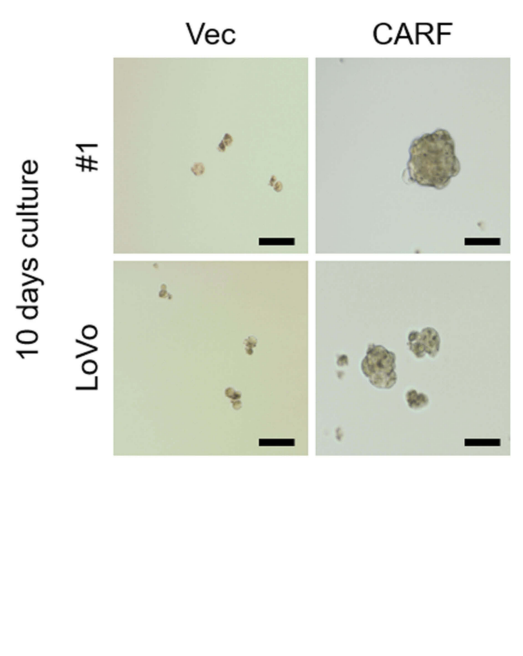

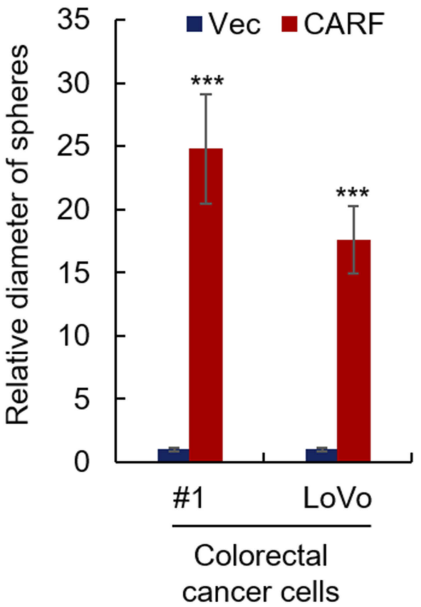

C

\#1

- Vec $\bullet$ CARF

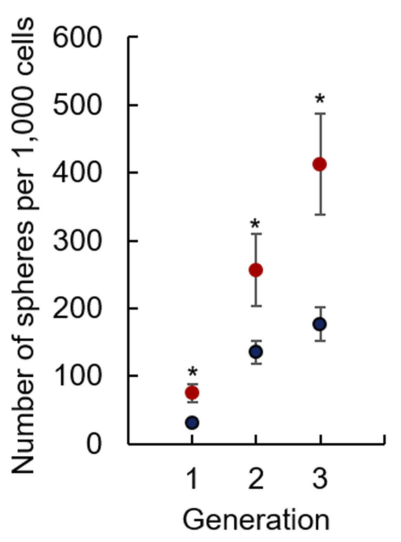

E

\#1

- Vehicle $\quad 5-F U$

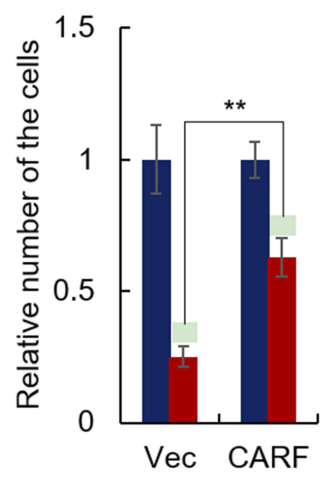

LoVo

- Vec • CARF

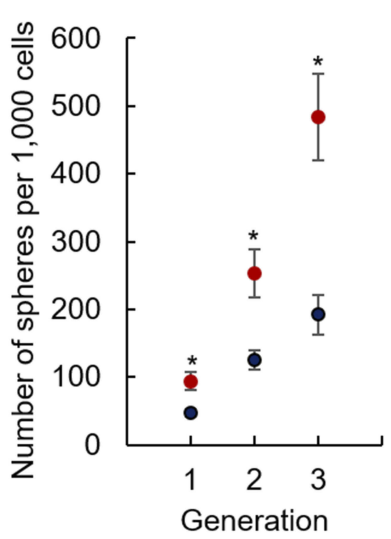

LoVo

- Vehicle $\quad 5-F U$

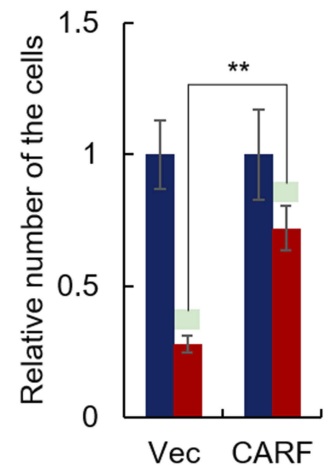

- Vec $\square \mathrm{CARF}$

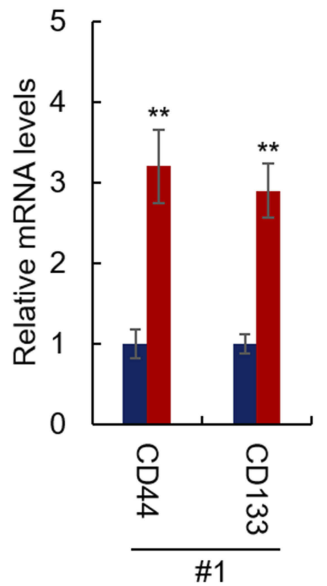

- Vec $-\mathrm{CARF}$

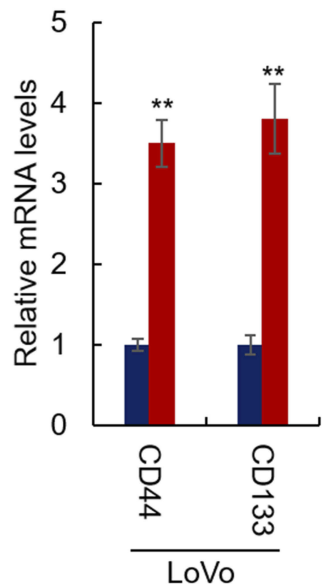

Figure 2 Ectopic expression of CARF promotes the stemness of colorectal cancer stem cells.

Notes: (A) The mRNA level of CARF in indicated cells was analyzed by qRT-PCR. (B) The diameter of the spheres from CARF-overexpressing cells and control cells was analyzed.

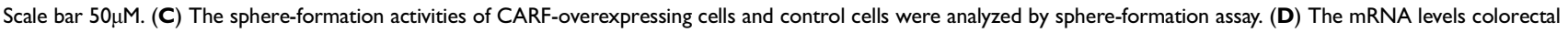
cancer stem cell markers in indicated cells were analyzed by qRT-PCR. (E) The sensitivity of the indicated cell to 5-FU was examined by CCK-8 assay. $(\mathbf{F})$ The tumorigenesis of indicated cells was analyzed by the xenograft model. The data are presented as mean \pm SD and representative of three independent experiments $(* P<0.05, * * P<0.01$, $* * * P<0.00 I)$. 
A
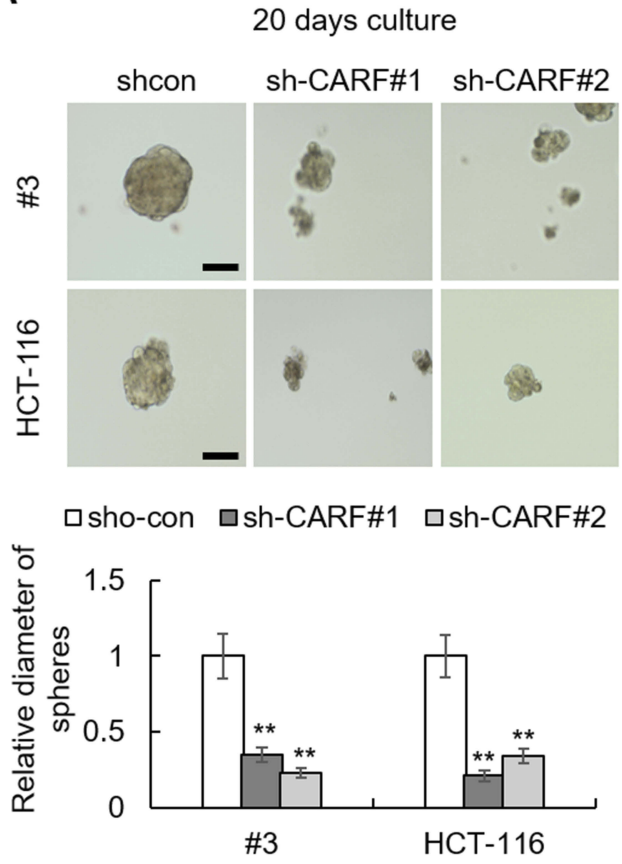

D
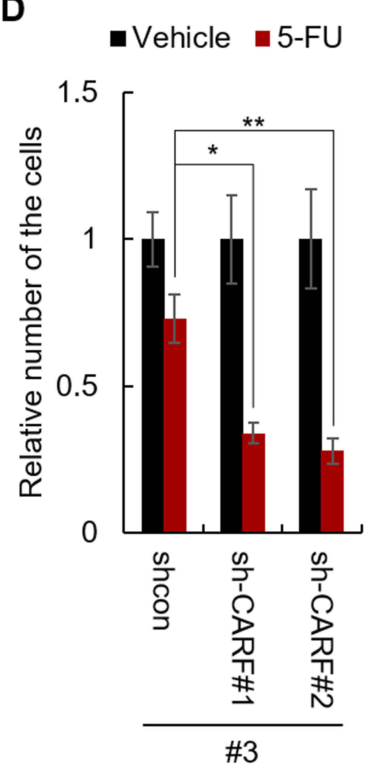

-Vehicle -5-FU

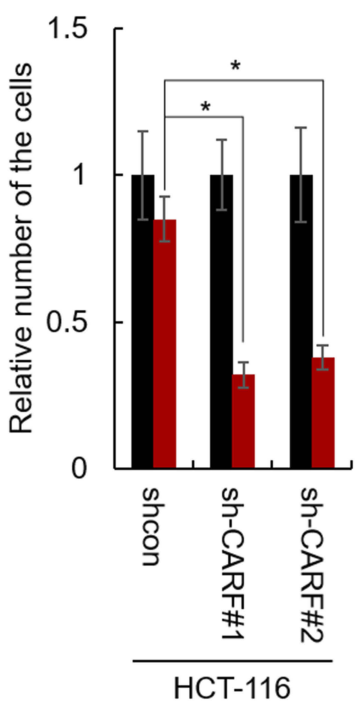

B

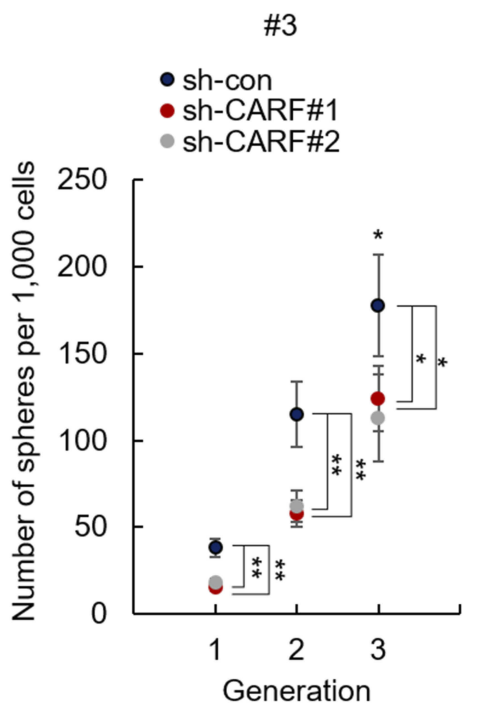

HCT-116

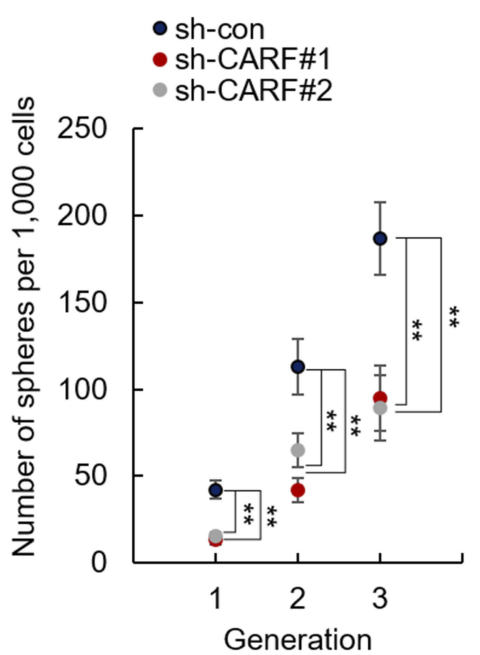

C
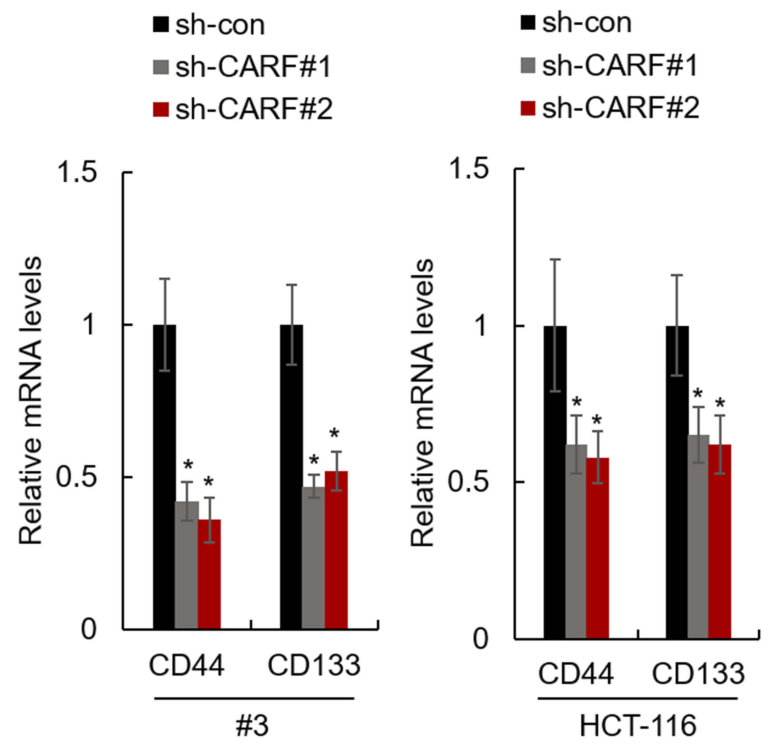

Figure 3 Knockdown of CARF inhibits the stemness of colorectal cancer stem cells.

Notes: (A) The diameter of the spheres from CARF-knockdown and control cells was analyzed. (B) The sphere-formation activities of indicated cells were analyzed by sphere-formation assay. (C) The mRNA levels colorectal cancer stem cell markers in indicated cells were analyzed by qRT-PCR. (D) The sensitivity of the indicated cell to 5 FU was examined by CCK-8 assay. The data are presented as mean \pm SD and representative of three independent experiments $(* P<0.05$, $* * P<0.01)$.

the protein level of MAPK8 and JUN were also upregulated in CARF-overexpressing colorectal cancer cells (Figure 4C). Moreover, we found that the mRNA levels the target genes of ERBB signaling pathway (FOSB and NFATC2) were significantly upregulated by CARF overexpression as identified by qRT-PCR and Western blot assay (Figure 4D and E). These results thus demonstrated that CARF activates the ERBB signaling pathway in colorectal cancer cells.

\section{ERBB Signaling Pathway Is Necessary For CARF Promoting Colorectal Cancer Stemness}

To confirm the critical role of ERBB signaling pathway in CARF promoting colorectal cancer stemness, we produced JUN-knockdown CARF-overexpressing cell lines (Supplementary Figure 3). In addition, the ERBB signaling 
A

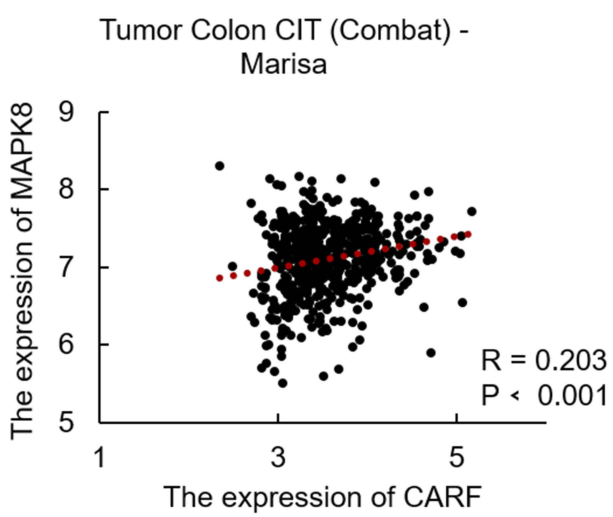

C

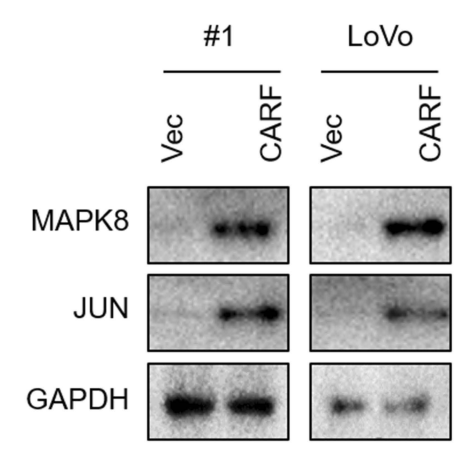

Tumor Colon CIT (Combat) -

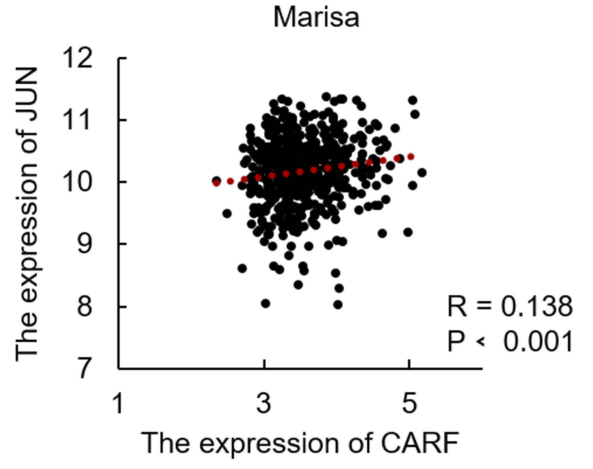

B
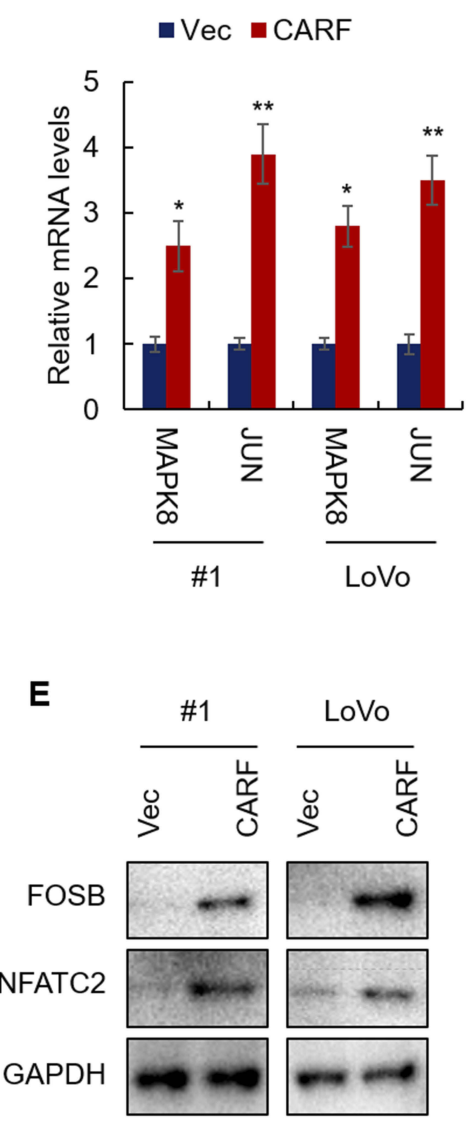

Figure 4 CARF activates the ERBB signaling pathway in colorectal cancer cells.

Notes: (A) The expression of CARF is positively correlated with MAPK8 and JUN. Gene expression data were downloaded from the R2 genomics analysis and visualization platform. Spearman correlation analysis was performed. (B) The mRNA levels of MAPK8 and JUN in indicated cells were analyzed by qRT-PCR. (C) The protein levels of MAPK8 and JUN in indicated cells were analyzed by Western blot. (D) The mRNA levels of FOSB and FATC2 in indicated cells were analyzed by qRT-PCR. (E) The protein levels of FOSB and FATC2 in indicated cells were analyzed by Western blot. The data are presented as mean \pm SD and representative of three independent experiments $(* P<0.05, * * P<0.01$, *** $P<0.001)$

was also inhibited by the JUK inhibitor (SP600125). As shown in Figure 5A, knockdown of JUN significantly decreased the CARF-induced increased expression of ERBB target genes (Figure 5A). In addition, knockdown of JUN significantly abolished the effect of CARF on sphereformation activities (Figure 5B and C). Furthermore, we found that CARF-induced increased expression of stem cell markers (CD44 and CD133) was significantly downregulated by JUN depletion (Figure 5D). As expected, similar results were obtained in SP600125-treated cells (Figure 5A-D). These results thus demonstrated that the ERBB signaling pathway is necessary for CARF promoting colorectal cancer stemness.

\section{CARF Activates The Transcription Of MAPK8 And JUN By Directly Binding To Their Promoters}

To further investigate the mechanisms underlying the effect of CARF on the transcription of MAPK8 and JUN, we performed luciferase reporter gene assay and ChIP assay to investigate whether CARF directly binds to the promoters of MAPK8 and JUN, as the fact that CARF is a transcription factor. As shown in Figure 6A, the results from luciferase reporter gene assay showed that CARF could activate the transcriptional function of the promoters of MAPK 8 and JUN. Moreover, as shown in Figure 6B, the results from the ChIP study 
A

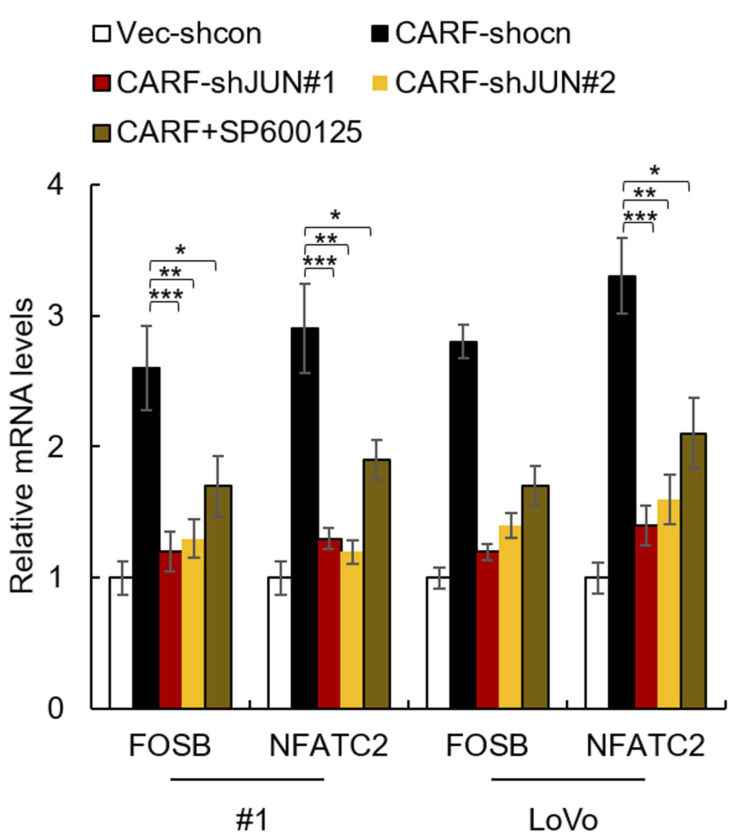

C

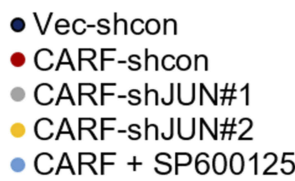

- Vec-shcon

- CARF-shJUN\#2

- CARF + SP600125
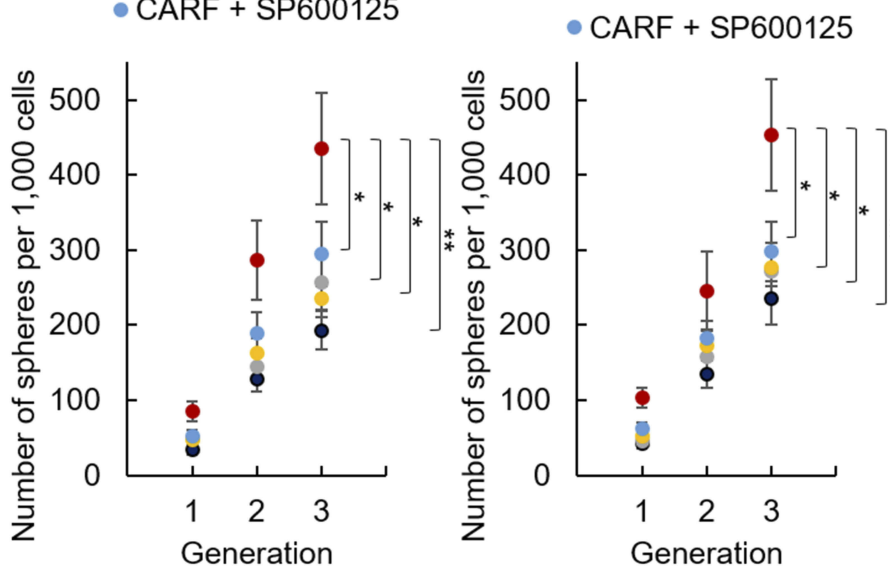

\section{B}

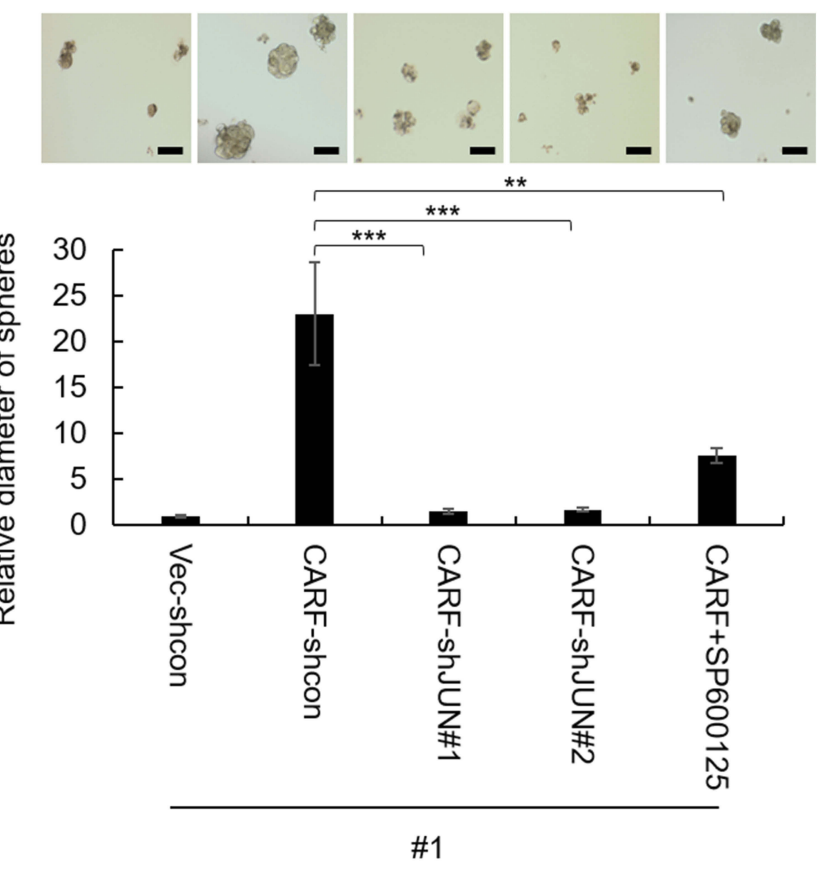

$\begin{array}{ll}\text { D } & \square \text { Vec-shcon } \\ \square \text { CARF-shJUN\#1 } & \square \text { CARF-shocn } \\ & \square \text { CARF+SP } 600125\end{array}$

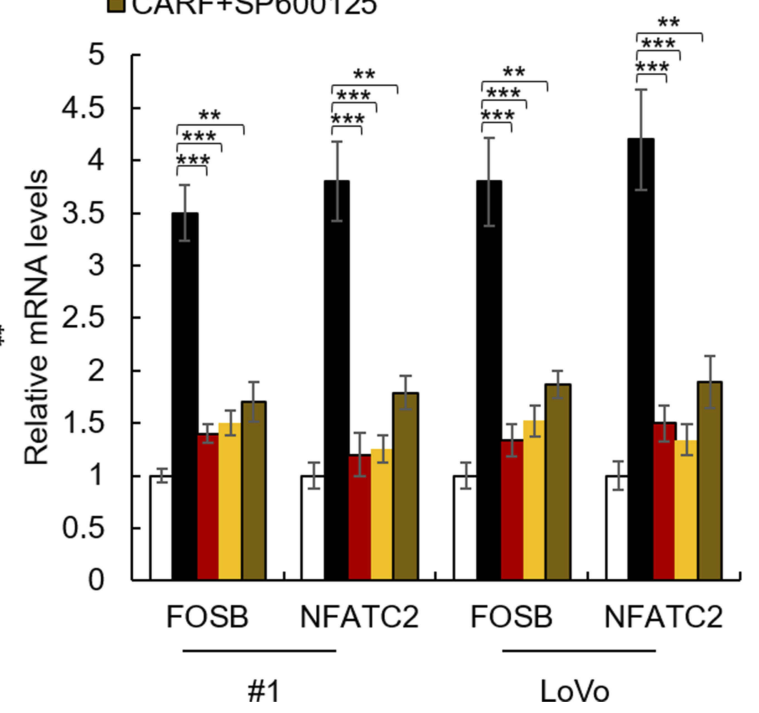

Figure 5 Knockdown of JUN abolished the effect of CARF on the stemness of colorectal cancer stem cells.

Notes: (A) The mRNA level of FOSB and NFATC2 in indicated cells was analyzed by qRT-PCR. (B) The diameter of the spheres from indicated cells and control cells was analyzed. Scale bar $50 \mu \mathrm{M}$ (C) The sphere-formation activities of indicated cells were analyzed by sphere-formation assay. (D) The mRNA levels colorectal cancer stem cell markers in indicated cells were analyzed by qRT-PCR. The data are presented as mean \pm SD and representative of three independent experiments $(* P<0.05$, $* * P<0.01$, $* * * P<0.001)$.

demonstrated that CARF could directly bind to the promoter of MAPK8 and JUN. Furthermore, we investigated the protein level of CARF in the nucleus and found that the nuclear level of CARF in significantly upregulated in CARF-overexpressing cells (Supplementary Figure 4), which confirmed the function of CARF in the nucleus. Collectively, the above results demonstrated that CARF aberrantly upregulates in colorectal cancer cells and promotes the maintenance of the stemness of colorectal cancer stem cell through the ERBB signaling pathway via directly binding to the promoter of MAPK8 and JUN (Figure 6C). 
A

口Vec a CARF

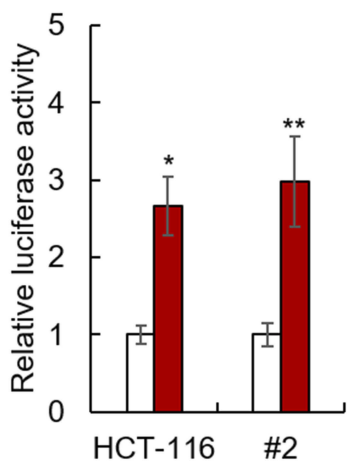

B

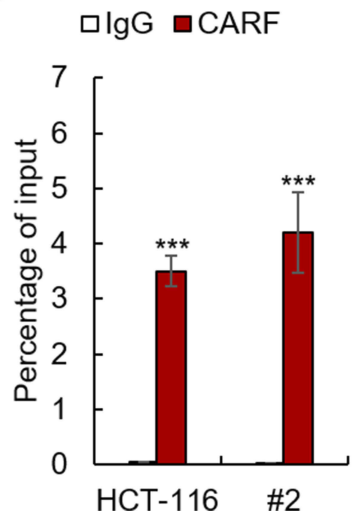

aVec $\square \mathrm{CARF}$

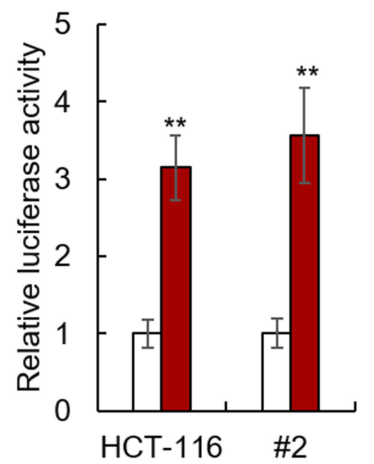

口IgG aCARF

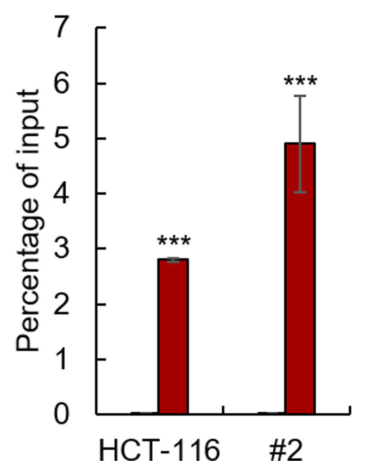

C

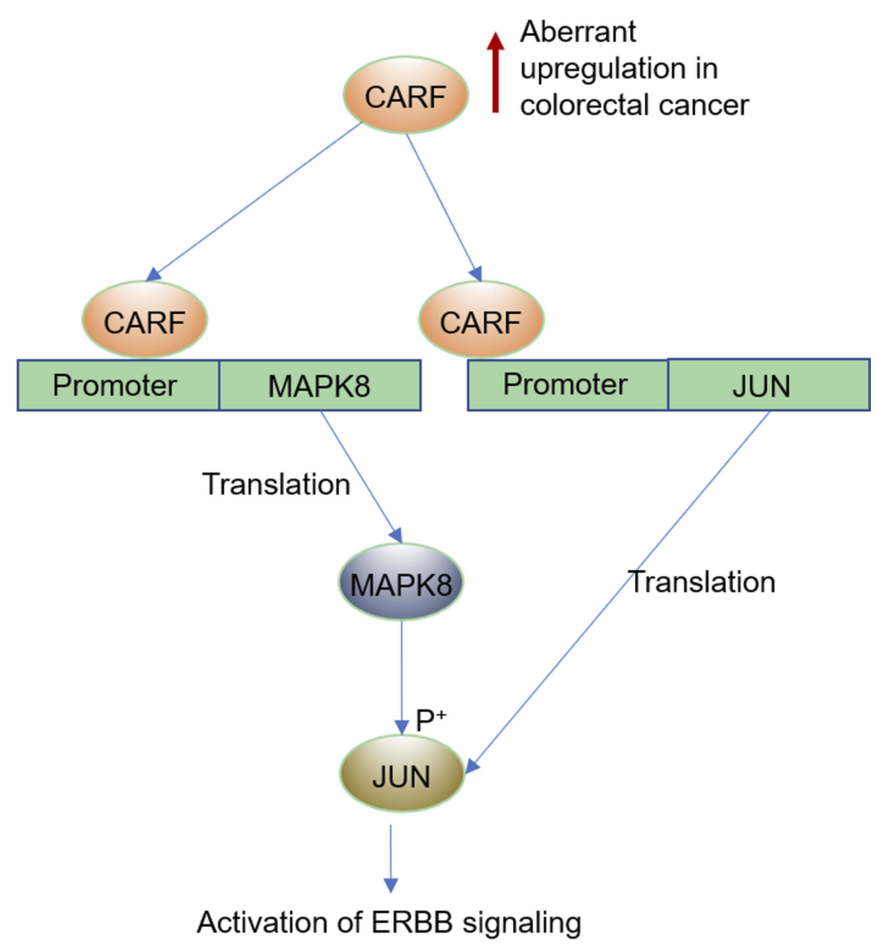

Figure 6 CARF activates the transcription of MAPK8 and JUN by directly binding to their promoters.

Notes: (A) The effects of CARF on the promoters of MAPK8 and JUN in indicated cells were examined by reporter gene assay. (B) The binding between CARF and the promoters of MAPK 8 and JUN in indicated cells was examined by ChIP study. (C) A model of the biological function of CARF in cancer stemness maintenance. The data are presented as mean $\pm S D$ and representative of three independent experiments $(* P<0.05, * * P<0.01, * * * P<0.00 I)$.

\section{Discussion}

In this study, we, for the first time, revealed the relationship between CARF, a calcium-responsive factor and cancer development. We showed that CARF aberrantly upregulates in colorectal cancer cells and promotes the maintenance of the stemness of colorectal cancer stem cell through the ERBB signaling pathway.

Colorectal cancer is a leading cause of cancer death, with its incidence increasing year by year. ${ }^{1}$ The development of precision medicine provides the chances to improve the therapeutic efficiency, ${ }^{4}$ however, our understanding of colorectal cancer initiation, progression and treatment resistance still needs to be improved.

Calcium ions $\left(\mathrm{Ca}^{2+}\right)$ are the most important second messenger in the human body which play multiple roles in fundamental cellular physiology, including gene expression, cell cycle, autophagy and apoptosis. ${ }^{5-8}$ Both extracellular and intracellular $\mathrm{Ca}^{2+}$ affect downstream effectors, such as transcription factors and kinases, altering the status of the cells $;{ }^{9-11}$ however, the mechanisms underlying the effect of $\mathrm{Ca}^{2+}$ signaling pathway on colorectal cancer have not been fully understood.

CARF is a transcription factor that was known as a $\mathrm{Ca}^{2+}$-responsive binding protein for the promoter of the gene encoding Bdnf, ${ }^{13-17}$ making a possible that CARF is a bridge between $\mathrm{Ca}^{2+}$ aberrant alteration and cancer initiation. Indeed, via bioinformatic analysis, we found that the upregulation of CARF is associated with poor prognosis of colorectal cancer patients (Figure 1A). Moreover, we found that CARF is upregulated in colorectal cancer tissues and colorectal cancer stem cells isolated from both primary cells and cell line cells (Figure 1B-D). Furthermore, the expression of CARF is positively correlated with CD44 and CD133 (Figure 1E). These results suggested that CARF is an oncogene in colorectal cancer. Next, genetic manipulation assay showed that the overexpression of CARF promotes the stemness of the colorectal cancer cells (Figure 2). Oppositely, the knockdown of CARF attenuates the stemness of colorectal cancer cells (Figure 3), which confirmed that CARF serves as an oncogene in colorectal cancer. 
Next, to reveal the mechanism underlying CARFinduced colorectal cancer stemness, we performed bioinformatic analysis and found that the expression of CARF is positively correlated with MAPK8 and JUN (Figure 4A-C), the most important factors in ERBB signaling pathway ${ }^{18}$, making the possibility that ERBB signaling mediates the CARF and colorectal cancer stemness as the fact that ERBB signaling pathway drives the stemness in colorectal cancer cells. ${ }^{19-21}$ Indeed, we next found that knockdown of JUN abolished the effect of CARF on the stemness of colorectal cancer cells (Figure 5), which confirmed the important role of ERBB signaling in this process. Moreover, we showed that CARF directly binds the promoter of MAPK8 and JUN as identified by luciferase reporter gene assay and ChIP-qPCR assay (Figure 6A and $\mathrm{B})$. Collectively, our study demonstrated that CARF is aberrantly upregulated in colorectal cancer cells and promotes the maintenance of the stemness of colorectal cancer stem cell through the ERBB signaling pathway via directly binding to the promoter of MAPK 8 and JUN (Figure 6C).

\section{Conclusion}

In summary, our studies revealed that CARF promotes colorectal cancer stemness by activating the ERBB signaling pathway. ERBB serves as the main downstream effector of CARF could be an efficient drug target for colorectal cancer caused by CARF aberrant expression.

\section{Author Contributions}

All authors contributed to data analysis, drafting and revising the article, gave final approval of the version to be published, and agree to be accountable for all aspects of the work.

\section{Disclosure}

The authors report no conflicts of interest in this work.

\section{References}

1. Siegel RL, Miller KD, Fedewa SA, et al. Colorectal cancer statistics, 2017. CA Cancer J Clin. 2017;67(3):177-193. doi:10.3322/caac.21395

2. Akhtar R, Chandel S, Sarotra P, Medhi B. Current status of pharmacological treatment of colorectal cancer. World J Gastrointest Oncol. 2014;6(6):177-183. doi:10.4251/wjgo.v6.i6.177

3. Van der Jeught K, Xu H-C, Li Y-J, Lu X-B, Ji. G. Drug resistance and new therapies in colorectal cancer. World J Gastroenterol. 2018;24 (34):3834-3848. doi:10.3748/wjg.v24.i34.3834
4. Tran NH, Cavalcante LL, Lubner SJ, et al. Precision medicine in colorectal cancer: the molecular profile alters treatment strategies. Ther Adv Med Oncol. 2015;7(5):252-262. doi:10.1177/175883401 5591952

5. Monteith GR, Prevarskaya N, Roberts-Thomson SJ. The calciumcancer signalling nexus. Nat Rev Cancer. 2017;17(6):367-380. doi:10.1038/nrc.2017.18

6. Cui C, Merritt R, Fu L, Pan Z. Targeting calcium signaling in cancer therapy. Acta Pharm Sin B. 2017;7(1):3-17. doi:10.1016/j.apsb.2016. 11.001

7. Xu M, Seas A, Kiyani M, Ji KSY, Bell HN. A temporal examination of calcium signaling in cancer- from tumorigenesis, to immune evasion, and metastasis. Cell Biosci. 2018;8:25. doi:10.1186/s13578-018-0223-5

8. Stewart TA, Yapa KT, Monteith GR. Altered calcium signaling in cancer cells. Biochim Biophys Acta. 2015;1848(10 Pt B):2502-2511. doi:10.1016/j.bbamem.2014.08.016

9. Hu Q, Deshpande S, Irani K, Ziegelstein RC. $[\mathrm{Ca}(2+)](\mathrm{i})$ oscillation frequency regulates agonist-stimulated NF-kappaB transcriptional activity. J Biol Chem. 1999;274(48):33995-33998. doi:10.1074/jbc. 274.48.33995

10. Cullen PJ. Calcium signalling: the ups and downs of protein kinase C. Curr Biol. 2003;13(18):R699-R701. doi:10.1016/j.cub.2003.08. 041

11. Smedler E, Uhlén P. Frequency decoding of calcium oscillations. Biochim Biophys Acta. 2014;1840(3):964-969. doi:10.1016/j. bbagen.2013.11.015

12. Zhu H, Zhang H, Jin F, et al. Elevated Orail expression mediates tumor-promoting intracellular $\mathrm{Ca} 2+$ oscillations in human esophageal squamous cell carcinoma. Oncotarget. 2014;5(11):3455-3471. doi:10. 18632/oncotarget.v5i11

13. Tao X, West AE, Chen WG, Corfas G, Greenberg ME. A calciumresponsive transcription factor, $\mathrm{CaRF}$, that regulates neuronal activity-dependent expression of BDNF. Neuron. 2002;33(3):383-395. doi:10.1016/S0896-6273(01)00561-X

14. Lyons MR, Chen LF, Deng JV, et al. The transcription factor calciumresponse factor limits NMDA receptor-dependent transcription in the developing brain. J Neurochem. 2016;137(2):164-176. doi:10.1111/ jnc. 13556

15. Agostino PV, Cheng RK, Williams CL, West AE, Meck WH. Acquisition of response thresholds for timed performance is regulated by a calcium-responsive transcription factor, CaRF. Genes Brain Behav. 2013;12(6):633-644. doi:10.1111/gbb.12059

16. Cheung CT, Singh R, Yoon AR, et al. Molecular characterization of apoptosis induced by CARF silencing in human cancer cells. Cell Death Differ. 2011;18(4):589-601. doi:10.1038/cdd.2010.129

17. Pfenning AR, Kim TK, Spotts JM, Hemberg M, Su D, West AE. Genome-wide identification of calcium-response factor (CaRF) binding sites predicts a role in regulation of neuronal signaling pathways. PLoS One. 2010;5(5):e10870. doi:10.1371/journal.pone.0010870

18. Arteaga CL, Engelman JA. ERBB receptors: from oncogene discovery to basic science to mechanism-based cancer therapeutics. Cancer Cell. 2014;25(3):282-303. doi:10.1016/j.ccr.2014.02.025

19. Szmida E, Karpiński P, Leszczynski P, et al. Aberrant methylation of ERBB pathway genes in sporadic colorectal cancer. J Appl Genet. 2015;56(2):185-192. doi:10.1007/s13353-014-0253-6

20. Hu YP, Venkateswarlu S, Sergina N, et al. Reorganization of ErbB family and cell survival signaling after Knock-down of ErbB2 in colon cancer cells. J Biol Chem. 2005;280(29):27383-27392. doi:10. 1074/jbc.M414238200

21. Filippi A, Ciolac OA, Ganea C, Mocanu MM. ErbB proteins as molecular target of dietary phytochemicals in malignant diseases. $J$ Oncol. 2017;2017:1532534. doi:10.1155/2017/1532534 


\section{Publish your work in this journal}

OncoTargets and Therapy is an international, peer-reviewed, open access journal focusing on the pathological basis of all cancers, potential targets for therapy and treatment protocols employed to improve the management of cancer patients. The journal also focuses on the impact of management programs and new therapeutic agents and protocols on patient perspectives such as quality of life, adherence and satisfaction. The manuscript management system is completely online and includes a very quick and fair peer-review system, which is all easy to use. Visit http://www.dovepress.com/ testimonials.php to read real quotes from published authors.

Submit your manuscript here: https://www.dovepress.com/oncotargets-and-therapy-journal 